\title{
Effect of Surface Functionalization on the Dielectric Properties of Polypropylene Aluminium Nitride Nanocomposites
}

\author{
Xinyu Wang, Thomas Andritsch, George Chen \\ Tony Davies High Voltage Laboratory \\ University of Southampton \\ Southampton, UK \\ xw2n14@soton.ac.uk
}

\begin{abstract}
Polymer nanocomposites can potentially provide many advantages and the interaction region between polymer and nanoparticles - the so-called interphase is usually considered to be responsible for the change of properties. In this work, nanoaluminium nitride powders with various surface states obtained by three different silane coupling agents (SCA) and an untreated powder, were blended with isotactic polypropylene. AC breakdown strength and dielectric spectroscopy were used to study the effect of different particle surfaces and polymerparticle interphases. The nano-aluminium nitride powder grafted with different organofunctional groups can provide a number of potential interactions with the matrix polymer. The results show that it can not only affect the dispersion state of nanoparticles but will provide different impacts on the dielectric properties of the bulk material. The hydrolysable groups of SCA, notably, can also influence the dielectric properties through altering the surface chemistry of nanoparticles.
\end{abstract}

Keywords—nanocomposites; interphase; polypropylene; nanoaluminium nitride; silane coupling agent; $A C$ breakdown; dielectric spectroscopy

\section{INTRODUCTION}

Due to the excellent dielectric properties and potential high operation temperature, polypropylene (PP) is considered to have great potential for renewable HVDC cable insulation material. However, the development and application of PP are limited by its relatively poor thermal conductivity [1]. Nanofillers with high thermal conductivity, such as aluminium nitride (AIN) have been reported can enhance the thermal conductivity of the base polymer [2]. In addition to the thermal conductivity, there has been a wide interest in nanodielectrics since it can potentially bring improved dielectric properties, such as increased breakdown strength and lower dielectric losses. This study focuses on the dielectric aspect of nanoAlN/polypropylene nanocomposites in regards of interphase properties. Based on recent studies [3, 4], the polymer-particle interphase region is believed to have a significant influence on the dielectric properties of nanocomposites. Thus, the proposed work is to investigate the influence of polymer-particle interphase by obtaining different particle surface states via different surface treatments.
The organofunctional group of SCA can interact with the base polymer by means of enhancing the compatibility between the inorganic particle and the organic polymer, but unlike polar polymer such as epoxy, it is hardly reported any SCA can build a strong interaction with a non-polar polymer like PP. In this study, SCA with methacrylate and octyl group were selected based on the different interaction with PP as it is reported that the methacrylate group can provide better compatibility [5]. In addition, octyl silanes with different hydrolysable groups were adopted in the proposed study, namely the methoxy and the ethoxy silane. Since few studies have investigated the influence of surface treatment with different SCA with different both organofunctional groups and hydrolysable groups, this work, therefore, can explore how the interphase affects dielectric properties of the bulk material depending on the different surface chemistry of nanoparticles.

\section{EXPERIMENTAL}

\section{A. Materials}

Three silane coupling agents with varying organofunctional and hydrolysable groups were used: The triethoxy (octyl) silane (C8-E) with a $\geq 97.5 \%$ quoted purity and 3(trimethoxysilyl) propyl methacrylate (MPTS-M) with a $98 \%$ quoted purity were purchased from Sigma-Aldrich; The trimethoxy (octyl) silane (C8-M) with a $97 \%$ quoted purity was obtained from Aladdin industrial Inc. The details of SCA used can be found in Table 1. The polymer matrix used in this work was isotactic polypropylene, which was obtained from Sigma Aldrich, with a melting temperature in the range of $160-165{ }^{\circ} \mathrm{C}$. The weight average molecular weight and the number average molecular weight are $\mathrm{Mw} \sim 340,000$ and $\mathrm{Mn}$ $\sim 97,000$, respectively. The nano-AIN was purchased from Aladdin industrial Inc. with a quoted average diameter of 50 $\mathrm{nm}$ and a quoted $99.9 \%$ metal-based purity.

\section{B. Surface functionalization of nano-AlN}

Toluene was selected as solvent since the anhydrous method was chosen in the present research. In addition, the condensation of SCA can be restricted when using hydroxyl free solvents like toluene, and this process cannot be controlled when the hydrolysis method is applied [6]. 


Chemical name and (denote)

To ensure the level of functionalization with each different SCA was performed to an equivalent extent, an estimation method was applied based on previous work [4], and the amounts of SCA applied are listed in Table 1. Here, $2 \mathrm{~g}$ of nano-AlN was added to $20 \mathrm{ml}$ of dry toluene. The nano-AlN/ dry toluene solution was sonicated for $30 \mathrm{~min}$ with a Hielscher UPS200S probe sonicator at $60 \%$ magnitude, and $0.5 \mathrm{~s}$ pulse in $1.0 \mathrm{~s}$ cycle, then stirred and sonicated for another $30 \mathrm{~min}$. After that, the mixture was poured into a $100 \mathrm{ml}$ evaporation flask. At the same time, the desired amount of SCA was dissolved in $20 \mathrm{ml}$ of dry toluene and added to the same flask. The nano-AlN/SCA/dry toluene mixture was then stirred at 120 rpm by a magnetic stirrer with a heater and heated to reflux at the boiling point of toluene for $4 \mathrm{~h}$. The resulting slurry was washed with $100 \mathrm{ml}$ of toluene three times $(300 \mathrm{ml}$ in total). Subsequently, centrifuging at $8000 \mathrm{rpm}$ was used to recover the nano-AlN during the washing operation.

\section{Blending}

Either 0.5 or $1.0 \mathrm{~g}$ of functionalized or untreated nano-AlN was dispersed in $20 \mathrm{ml}$ of toluene (60 min of sonication with stirring halfway through was applied to the untreated powder) and then added to $100 \mathrm{ml}$ of boiling xylene with 9.5 or $9.0 \mathrm{~g}$ of dissolved PP. The resulting mixture was stirred vigorously until it becomes a rubber-like chunk. After that, the material was cut into $\sim 5 \mathrm{~mm}$ cubes and dried for $24 \mathrm{~h}$ in a fume cupboard. After the majority of solvent was evaporated, the nanocomposite cubes were pressed into thick sheets at $180{ }^{\circ} \mathrm{C}$ and then put into a vacuum oven to evaporate the residual solvent for 3 days at $80{ }^{\circ} \mathrm{C}$. In the end, the nanocomposite was pressed into desired shapes for different tests. A total of nine dielectric systems were prepared, including 0 wt. \%, 5 wt. \% and $10 \mathrm{wt}$. \% of loading ratios with three different surface functionalized fillers and one untreated powder as reference. The dielectric systems were denoted as "SCA-loading ratio" such that e.g. "MPTS-M-5" is representative of the 5 wt. \% of MPTS-M functionalized AIN/PP nanocomposite. The unfilled and untreated AlN/PP systems were denoted as PP (0 wt. \%), PP-5 (5 wt. \%) and PP-10 (10 wt. \%).

\section{Characterisation}

Thermogravimetric analysis (TGA) was used by heating 5 mg of the sample from $100{ }^{\circ} \mathrm{C}$ to $900{ }^{\circ} \mathrm{C}$ at the heating rate of $10{ }^{\circ} \mathrm{C} / \mathrm{min}$ in nitrogen to verify the surface functionalization and to $600{ }^{\circ} \mathrm{C}\left(20^{\circ} \mathrm{C} / \mathrm{min}\right)$ in air to verify the loading ratio of nanocomposites. Powders in toluene were isothermally treated at $110.6{ }^{\circ} \mathrm{C}$ in $\mathrm{N}_{2}(1 \mathrm{~h})$ to evaporate the solvent before analysis.

AC breakdown testing (ASTM D149 standard) was performed on $0.12 \mathrm{~mm}$ thick samples placed between two ball electrodes. The test cell was immersed in silicone oil to prevent flashover. The AC voltage with $500 \mathrm{~V} / \mathrm{s}$ ramp rate was applied to sample until breakdown. 20 points for each system were obtained, and the results were analysed by Weibull statistics.

Samples for scanning electron microscopy (SEM) were treated by an etching procedure outlined in [7]. They were then examined by a ZEISS field emission SEM at $20 \mathrm{kV}$, after gold coating with an Emitech K550X sputter coater.

Dielectric spectroscopy was carried out on $0.21 \mathrm{~mm}$ thick samples clamped by two $32 \mathrm{~mm}$ diameter electrodes. The real and imaginary permittivity were measured at $90{ }^{\circ} \mathrm{C}$ from 0.1 $\mathrm{Hz}$ to $100 \mathrm{kHz}$ using a Solartron 1296 interface with a Schlumberger SI 1260 impedance analyser.

\section{RESULTS AND DISCUSSION}

\section{A. TGA: verification of functionalization and loading ratio}

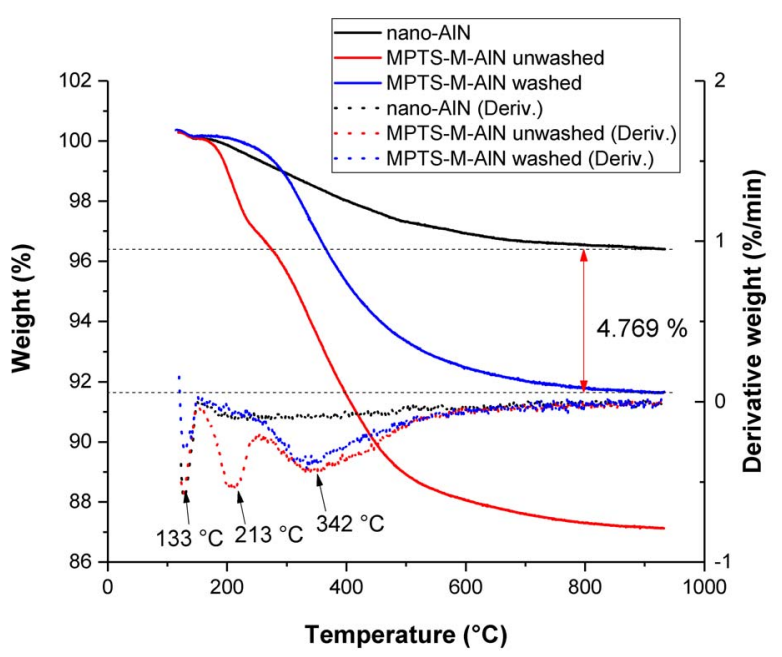

Fig. 1. the TGA results of as-received powder, and unwashed and washed nano-AIN after silane functionalized by MPTS-M. 
(a)

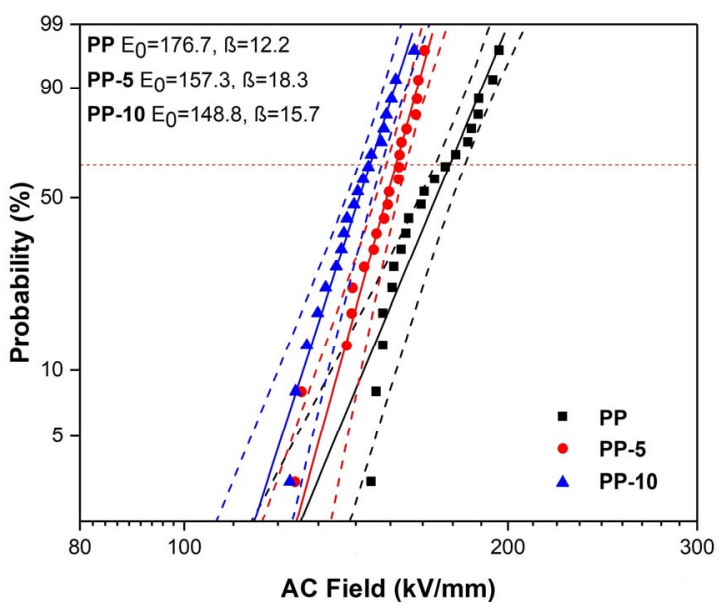

(c)

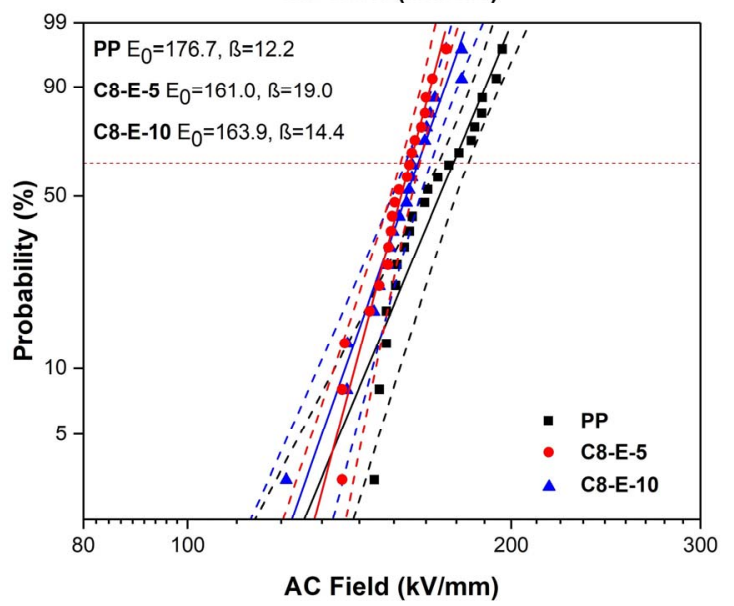

(b)

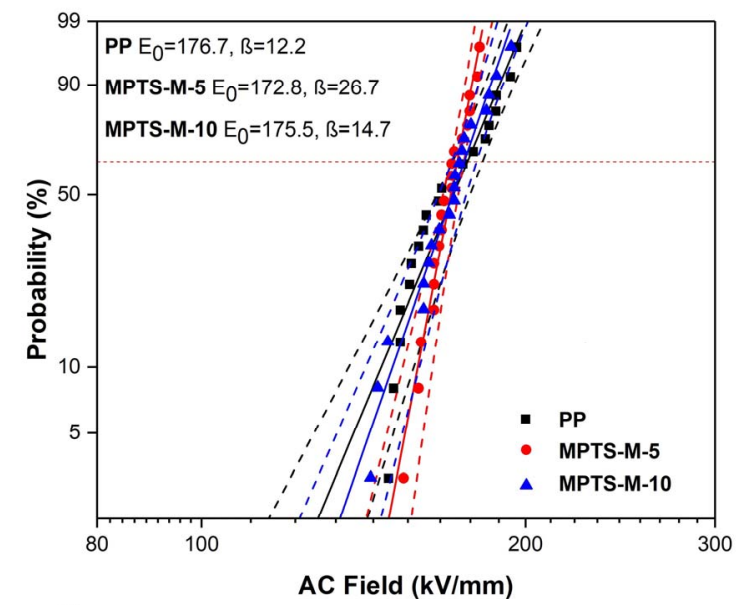

(d)

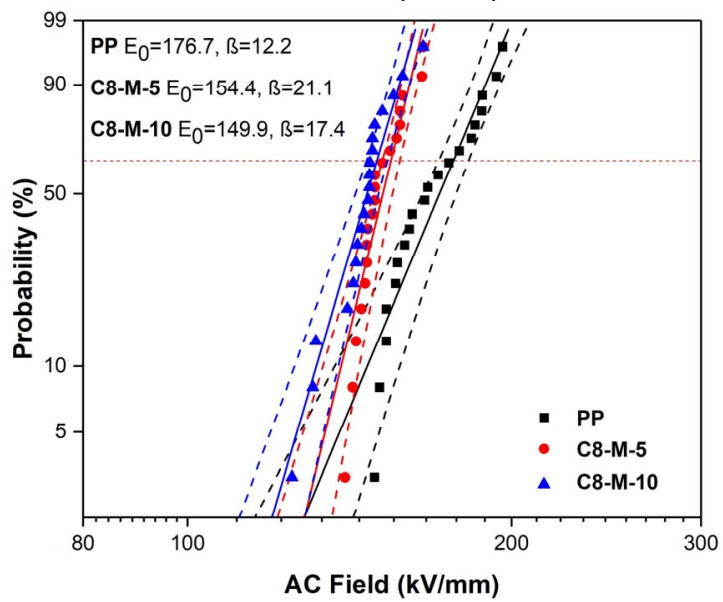

Fig. 2. the AC breakdown strengths of (a) untreated nano-AlN/PP and (b) MPTS-M, (c) C8-E and (d) C8-M functionalized nano-AlN/PP systems, Weibull parameters are listed in respective figures.

It can be seen from Fig. 1, the MPTS-M silane functionalized nano-AIN has $4.769 \%$ more weight loss than the untreated powder. The results for C8-M and C8-E were omitted due to the similarity of curves obtained. The additional weight losses were $4.681 \%$ and $3.692 \%$, respectively. From the derivative curve, the peaks at $213{ }^{\circ} \mathrm{C}$ and $342{ }^{\circ} \mathrm{C}$ are assigned to the weight loss of un-grafted and grafted MPTS-M. The peak at $133{ }^{\circ} \mathrm{C}$ is assigned to the loss of bonded water.

The TGA results show that the weights remain after heated to $600{ }^{\circ} \mathrm{C}$ of different nanodielectrics systems are: PP $(-0.799$ \%); PP-5 (4.929 \%); PP-10 (10.570 \%); MPTS-M-5 (4.076\%); MPTS-M-10 (9.452\%); C8-M-5 (4.864\%); C8-M-10 (9.223 \%); C8-E-5 (5.133\%); C8-E-10 (8.973\%). All 5 wt. \% samples have a real loading ratio of $4.6 \pm 0.6 \mathrm{wt} . \%$ and all $10 \mathrm{wt} . \%$ samples have a real loading ratio of $9.8 \pm 0.9$ wt. $\%$.

\section{B. AC breakdown and SEM}

From the AC breakdown strength results of PP-5 and PP-10 and the control group (PP) shown in Fig. 2 (a), the breakdown strengths of untreated nano-AlN/PP systems are lower than the breakdown strength of unfilled PP. It is noted that there is a decreasing trend when increasing the loading ratio from $5 \mathrm{wt}$. $\%$ to 10 wt. $\%$. This result could be explained by the defects and imperfections that are introduced by large agglomerations ( $>5 \mu \mathrm{m}$, in Fig. 4 (a)) in untreated nano-AlN/PP systems [8].
However, systems contain MPTS-M functionalized nanofiller (Fig. 2 (b)) show an increase in AC breakdown strength when compared to untreated nano-AlN/PP systems (Fig. 2 (a)). From the SEM result shown in Fig. 4 (a) and (b), no obvious improvement in the dispersion can be seen (the SEM results of 10 wt.\% were omitted due to the similar trend). From Fig. 4 (b), (c) and (d), the dispersion state of both $\mathrm{C} 8$ functionalized filler/PP systems appears to be better than the MPTS-M functionalized nano-AIN/PP system. But, counter-intuitively, both $\mathrm{C} 8$ functionalized fillers/PP systems show a decreased AC breakdown strength when compared to the unfilled PP (Fig. 2 (c) and (d)) while MPTS-M functionalized nano-AlN/PP systems have comparable AC breakdown strength to the unfilled PP (Fig. 2 (b)). This results may indicate, in the present study, the dispersion state of nano-filler play secondary order in determining AC breakdown strength [8]. The different interphase properties provided by different surface states of nanoparticles seems to play the significant role here.

In addition, the different $\mathrm{AC}$ breakdown results of $\mathrm{C} 8 \mathrm{-M}$ and C8-E functionalized nano-AlN/PP systems in Fig. 2 (c) and (d) indicate that the hydrolysable groups of SCA may also have effects on dielectric properties. It can be related to the different hydrolysis rate or grafting reaction rate during the silane functionalization. Further study is needed for both organofunctional and hydrolysable parts of SCA to come to definite conclusions. 

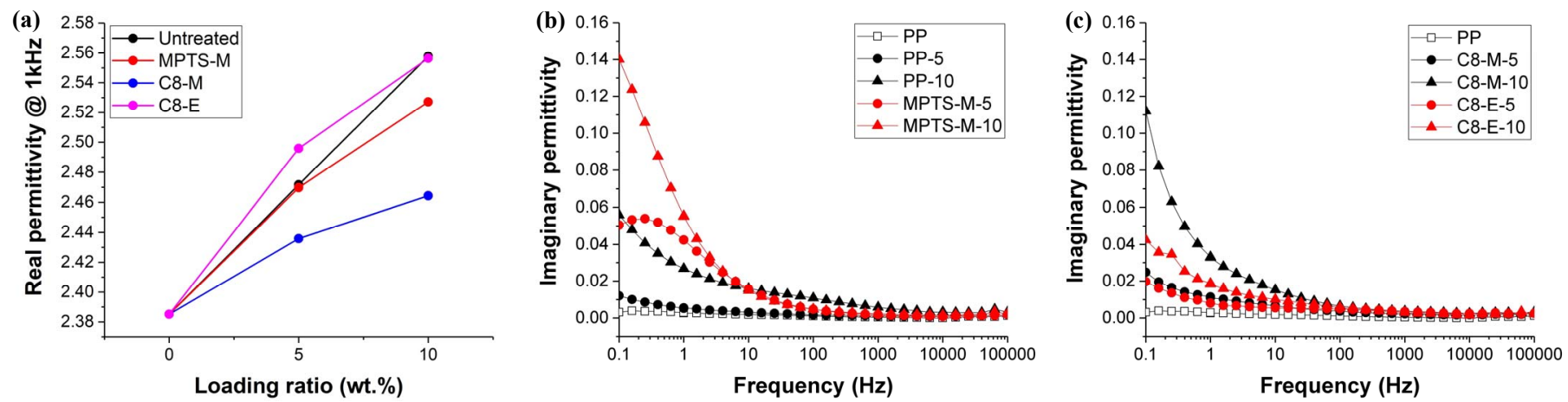

Fig. 3. (a) the real permittivities of each system of different loading ratio at $1 \mathrm{kHz}$, and the imaginary permittivities of (b) untreated nano-AIN/PP and MPTS-M functionalized nano-AlN/PP systems, (c) C8-E and C8-M functionalized nano-AlN/PP systems at $90{ }^{\circ} \mathrm{C}$.

\section{Dielectric spectroscopy}

The real permittivity of each system as a function of loading ratio at $1 \mathrm{kHz}$ is plotted in Fig. 3 (a). The real permittivity shows an increasing trend when the loading ratio increases. Both organofunctional and hydrolysable group seem to have a different impact on the real permittivity.

From Fig. 3. (b) and (c), MPTS-M functionalized nanoAlN/PP systems show the highest losses at low-frequency range $(<1 \mathrm{~Hz})$. It could be explained by the high polarity of methacrylate functional group grafted on the particle surface. However, comparable high losses of C8-M (non-polar) functionalized nano-AlN/PP systems at low-frequency range cannot support this conclusion. C8-E functionalized nanoAlN/PP systems show the best performance among all dielectric systems, which is not surprising and consistent with other work [2]. The non-polar structure of C8 functional groups grafted on the particle surface could be the dominant factor, which displaced the hydroxyl groups on the particle surface. The contradict results of $\mathrm{C} 8 \mathrm{-M}$ reveal the surface chemistry complexity of the silane functionalized nano-AIN. Further work related to formulating and characterising the interphase region between nano-filler and matrix (in surface chemistry aspect) is necessary.

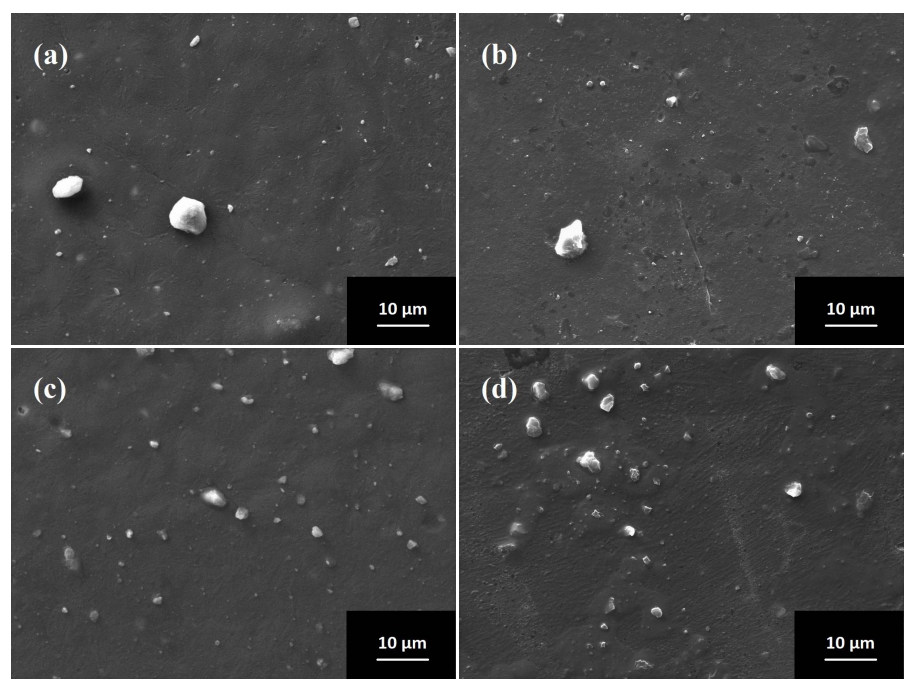

Fig. 4. the SEM images of (a) PP-5, (b) MPTS-M-5, (c) C8-M-5, (d) C8-E-5.

\section{Conclusions}

Polypropylene-based dielectric systems with four different nano-AlN fillers that have different surface states have been prepared to understand how the particle surface chemistry affects the dielectric properties of nanodielectrics.

The AC breakdown and the SEM results show that the surface chemistry of the filler, or the interphase properties, can be the dominant factor in determining AC breakdown strength, rather than the dispersion state of nano-fillers. From dielectric spectroscopy data, it can be concluded that, when considering the silane functionalization, the organofunctional group and the hydrolysable group can result in different dielectric responses of nanocomposites, by altering the surface states of nanoparticles. It is worth to note that although the hydrolysable group will be hydrolysed during silane functionalization, it can also affect the surface chemistry of nano-fillers by controlling the reaction mechanism, such as the grafting rate of SCA.

\section{REFERENCES}

[1] X. Huang, P. Jiang, and T. Tanaka, "A review of dielectric polymer composites with high thermal conductivity," IEEE Electrical Insulation Magazine, vol. 27, no. 4, pp. 8-16, 2011.

[2] Y. Zhou, J. Hu, X. Chen, F. Yu, and J. He, "Thermoplastic polypropylene/aluminum nitride nanocomposites with enhanced thermal conductivity and low dielectric loss," IEEE Transactions on Dielectrics and Electrical Insulation, vol. 23, no. 5, pp. 2768-2776, 2016.

[3] M. Roy, J. Nelson, R. MacCrone, L. S. Schadler, C. Reed, and R. Keefe, "Polymer nanocomposite dielectrics-the role of the interface," IEEE transactions on dielectrics and electrical insulation, vol. 12, no. 4, pp. 629-643, 2005.

[4] T. Andritsch, "Epoxy Based Nanodielectrics for High Voltage DC Applications: Synthesis, Dielectric Properties and Space Charge Dynamics," PhD Thesis, TU Delft, 2010.

[5] Y. Xie, C. A. S. Hill, Z. Xiao, H. Militz, and C. Mai, "Silane coupling agents used for natural fiber/polymer composites: A review," Composites Part A: Applied Science and Manufacturing, vol. 41, no. 7, pp. 806-819, 2010.

[6] C. Yeung, "Spectroscopic analysis of nanodielectric interfaces," $\mathrm{PhD}$ Thesis, University of Southampton, 2013.

[7] I. L. Hosier, M. Praeger, A. S. Vaughan, and S. G. Swingler, "The Effects of Water on the Dielectric Properties of Aluminum-Based Nanocomposites," IEEE Transactions on Nanotechnology, vol. 16, no. 4, pp. 667-676, 2017.

[8] Z. Li, K. Okamoto, Y. Ohki, and T. Tanaka, "Role of nano-filler on partial discharge resistance and dielectric breakdown strength of microAl 2 O 3/epoxy composites," in 9th International Conference on Properties and Applications of Dielectric Materials (ICPADM), pp. 753-756, 2009. 